\title{
BICEP2/SPUD: searching for inflation with degree scale polarimetry from the South Pole
}

Hien Trong Nguyen, John Kovac, Peter Ade, Randol Aikin, Steve Benton, et al.

Hien Trong Nguyen, John Kovac, Peter Ade, Randol Aikin, Steve Benton, James Bock, Justus Brevik, John Carlstrom, Darren Dowell, Lionel Duband, Sunil Golwala, Mark Halpern, Matthew Hasslefield, Kent Irwin, William Jones, Jonathan Kaufman, Brian Keating, Chao-Lin Kuo, Andrew Lange, Tomotake Matsumura, Barth Netterfield, Clem Pryke, John Ruhl, Chris Sheehy, Rashmi Sudiwala, "BICEP2/SPUD: searching for inflation with degree scale polarimetry from the South Pole," Proc. SPIE 7020, Millimeter and Submillimeter Detectors and Instrumentation for Astronomy IV, 70201F (21 July 2008); doi: 10.1117/12.787997

Event: SPIE Astronomical Telescopes + Instrumentation, 2008, Marseille, France 


\title{
BICEP2/SPUD: Searching for inflation with degree scale polarimetry from the South Pole
}

\author{
Hien Trong Nguyen ${ }^{*, a}$, John Kovac ${ }^{\mathrm{b}}$, Peter Ade ${ }^{\mathrm{c}}$, Randol Aikin ${ }^{\mathrm{b}}$, Steve Benton ${ }^{\mathrm{d}}$, Jamie Bock ${ }^{\mathrm{a}}$, Justus \\ Brevik $^{\mathrm{b}}$, John Carlstrom ${ }^{\mathrm{e}}$, Darren Dowell ${ }^{\mathrm{a}}$, Lionel Duband ${ }^{\mathrm{f}}$, Sunil Golwala ${ }^{\mathrm{c}}$, Mark Halpern ${ }^{\mathrm{g}}$, \\ Matthew Hasselfield ${ }^{\mathrm{g}}$, Kent Irwin ${ }^{\mathrm{h}}$, William Jones ${ }^{\mathrm{a}}$, Jonathan Kaufman ${ }^{\mathrm{i}}$, Brian Keating ${ }^{\mathrm{i}}$, Chao-Lin \\ $\mathrm{Kuo}^{\mathrm{j}}$, Andrew Lange ${ }^{\mathrm{b}}$, Tomotake Matsumura ${ }^{\mathrm{b}}$, Barth Netterfield ${ }^{\mathrm{c}}$, Clem Pryke ${ }^{\mathrm{e}}$, John Ruhl ${ }^{\mathrm{k}}$, Chris \\ Sheehy ${ }^{\mathrm{e}}$, Rashmi Sudiwala ${ }^{\mathrm{d}}$ \\ ${ }^{a}$ Jet Propulsion Laboratory, California Institute of Technology, USA; \\ ${ }^{\mathrm{b}}$ California Institute of Technology, USA; \\ ${ }^{\mathrm{c}}$ Cardiff University, UK; \\ ${ }^{\mathrm{d}}$ University of Toronto, CANADA; \\ ${ }^{\mathrm{e}}$ University of Chicago, USA; \\ ${ }^{\mathrm{f}}$ Commissariat 'a l'’Energie Atomique, Grenoble, France; \\ ${ }^{\mathrm{g}}$ University of British Columbia, CANADA; \\ ${ }^{\mathrm{h}}$ National Institute of Standards and Technology, Boulder, USA; \\ ${ }^{\mathrm{i}}$ University of California, San Diego, USA; \\ ${ }^{\mathrm{j}}$ Stanford University, USA; \\ ${ }^{\mathrm{k}}$ Case Western University, USA;
}

\begin{abstract}
BICEP2/SPUD is the new powerful upgrade of the existing BICEP1 experiment, a bolometric receiver to study the polarization of the cosmic microwave background radiation, which has been in operation at the South Pole since January 2006. BICEP2 will provide an improvement up to 10 times mapping speed at $150 \mathrm{GHz}$ compared to BICEP1, using the same BICEP telescope mount. SPUD, a series of compact, mechanically-cooled receivers deployed on the DASI mount at the Pole, will provide similar mapping speed in to BICEP2 in three bands, 100, 150, and $220 \mathrm{GHz}$. The new system will use large TES focal plane arrays to provide unprecedented sensitivity and excellent control of foreground contamination.
\end{abstract}

Keywords: CMB, polarization, bolometer, TES, south pole

\section{INTRODUCTION}

The subject of the cosmic microwave background (CMB) needs no further introduction at this year's SPIE (Marseille, 2008). Thanks to a number of experiments (Boomerang, DASI, WMAP, ACBAR, etc.), we have now learnt that the combined power spectrum - over a wide range of angular scale, from the entire sky down to the size of few arc seconds - can be described by no more than 5 cosmological parameters[1]. The Universe is found to be essentially isotropic and nearly flat. These two features are remarkably consistent with the theory of inflation.

It's important to point out that although the CMB was discovered more than 40 years ago, these high quality spatial data have been attained only recently thanks to concerted efforts made over the years by experimentalists from all over the world. The key factor in realizing these exquisitely detailed maps is the dramatic improvement of detector sensitivity that started to happen only over the last decade.

An electromagnetic field such as the $\mathrm{CMB}$ can be completely described by four fundamental quantities, these are the intensity, frequency distribution, spatial distribution and polarization. We have measured the first three quantities; each of these three quantities has in turn yielded information of fundamental significance about the nature of the Universe.

* email: hien.t.nguyen@jpl.nasa.gov; phone:1-818-354-0560; fax:1-818-354-8895

Millimeter and Submillimeter Detectors and Instrumentation for Astronomy IV edited by William D. Duncan, Wayne S. Holland, Stafford Withington, Jonas Zmuidzinas Proc. of SPIE Vol. 7020, 70201F, (2008) - 0277-786X/08/\$18 - doi: 10.1117/12.787997 
The last quantity, polarization, has so far remained to be the most challenging to characterize. One of reasons why this type of measurement has been carried out to a meaningful degree only over the last few years, perhaps, is the fact that the polarization signal is exceedingly small. And so far this proves to be yet the biggest challenge experimentally, at the same time it promises to bear the most exciting consequence scientifically.

Theory firmly predicts that inflation should create a cosmic gravitational wave background in the earliest moments of the Big Bang. The amplitude of these gravity waves measures the energy scale of inflation, and is thus highly model dependent. These gravitational waves then later interact with the scattering of CMB photons during recombination and reionization, leaving unique spatial pattern in the CMB polarization. In particular, a detection of CMB B-mode polarization is the definitive verification of inflation at work.

It had taken more than 2 decades to improve the detector sensitivity to detect temperature anisotropy at a level of a few parts in a thousand to a few parts in a hundred thousand to finally reveal the minute spatial structure. In order to detect the polarization signal from inflation, it is expected that a similar jump of at least another three orders of magnitude improvement in instrument sensitivity may be required. The good news is that today bolometer sensitivity, defined here as the time required to map a region of sky to a given sensitivity level, has significantly exceeded the Moore's law exponential growth rate for computer speed and memory. Another piece of good news is that if the power spectrum of the spatial fluctuations has a significant red tilt, as seen in a number of recent experiments ( $n=0.96$ as shown by WMAP), then in the simplest inflationary models, the polarization signal would be at the level of characterized by the ratio of tensor-to-scalar, $r$, of around 0.1 , a level which should be detectable with the next generation of instruments.

The 2006 NSF/NASA/DOE Task Force on CMB explicitly calls out, "at our highest priority, we recommend a phased program to measure the large-scale CMB polarization signal expected from inflation."[2] The BICEP2/SPUD experiment directly addresses this recommendation. BICEP2/SPUD is designed to be a powerful upgrade of the BICEP1 experiment[3][4] .

BICEP2/SPUD aims to ultimately detect the signature of inflation for $r<0.01$, as targeted by the CMB task force. In doing so, we expect to detect gravitational lensing of CMB polarization on scales $\sim 1$ degree, and to characterize the Galactic foregrounds in the cleanest field of the sky.

We plan to accomplish this goal by increasing the mapping speed of each of the new BICEP2/SPUD receivers up to 10 times compared to BICEP1. And we'll do this in three frequency bands, 100, 150 and $220 \mathrm{GHz}$, in order to provide excellent control of systemmatics and foregrounds.

\section{THE FOREGROUNDS AND SKY COVERAGE}

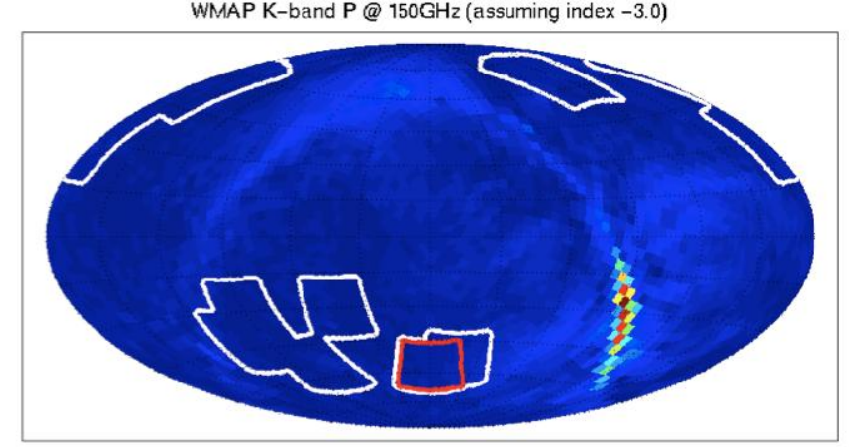

Color range 0 to $4 \mu \mathrm{K}$

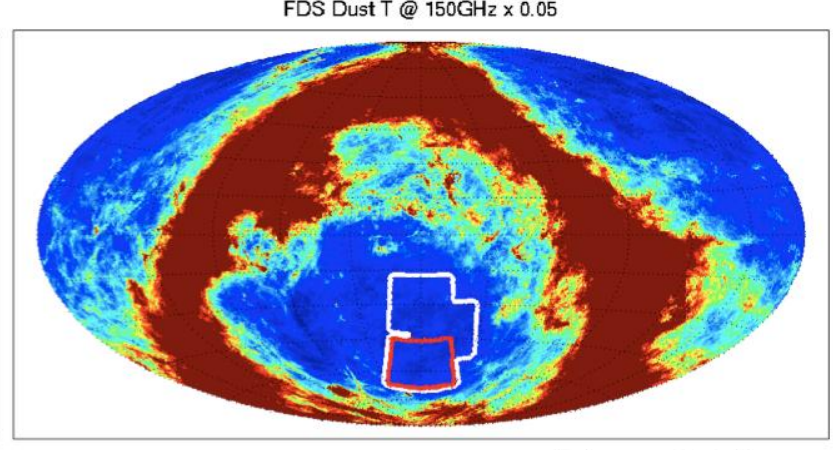

Color range 0 to $4 \mu \mathrm{K}$

Figure 1. The BICEP field in the southern sky, shown here in red outline, is remarkably free of Galactic polarized emission. The left panel shows an estimate of the level of polarized emission from galactic synchrotron radiation, based on WMAP measurements in K band, extrapolated to $150 \mathrm{GHz}$ assuming a synchrotron spectral index of -3 . The right panel shows the FDS model for thermal dust emission at $150 \mathrm{GHz}$ (based on IRAS and DIRBE measurements) multiplied by a nominal polarization fraction of 5\%. Other fields with equally low levels of emission in each template map compared to the BICEP field are outlined in white. The BICEP field is among the cleanest in the sky with respect to both foreground templates, but is particularly free from dust which exhibits far greater contrast. Low dust emission in this field may push the effective foreground minimum frequency as high as $150 \mathrm{GHz}$. 
The sensitive measurement that we are planning to make will require careful attention to foregrounds. The sources of foreground we currently consider come from galactic polarized emission and E to B mixing from gravitational lensing. The later is less significant at large angular scale and thus can be mitigated by choosing a relatively large area of sky. The former is greatly reduced if choosing a relatively clean region of sky. The angular power spectrum of dust emission at $\mathrm{l}=30-100$ in the Southern Hole is more than 100 times lower than the sky median. Assuming a 5\% polarization fraction, the B-mode from dust emission at $150 \mathrm{GHz}$ is equivalent to $\mathrm{r} \sim 0.02$, more than 10 times below current limits. Synchrotron B-mode in the Southern Hole is too faint to be well constrained by existing data; the WMAP $23 \mathrm{GHz}$ signal in this region only marginally exceeds the noise floor, but implies a level no greater than the dust foreground at 150 $\mathrm{GHz}$, assuming a spectral index -3.0. The Southern Hole thus offers a window on the extragalactic sky in which Galactic confusion could reasonably be expected to be below $\mathrm{r} \sim 0.03$ at $150 \mathrm{GHz}$ in the range $50<1<150$.

Our strategy is to deeply integrate $2 \%$ of the sky ( 800 square degree) on the cleanest hole in the southern sky, which is accessible from our South Pole observing platform. Our plan is thus phased. BICEP2 will first make the most sensitive measurements possible at $150 \mathrm{GHz}$. After BICEP2 we will follow with a series of receivers operating at 100 and 220 $\mathrm{GHz}$ to both improve sensitivity and to distinguish $\mathrm{CMB}$ polarization from Galactic foreground emission.

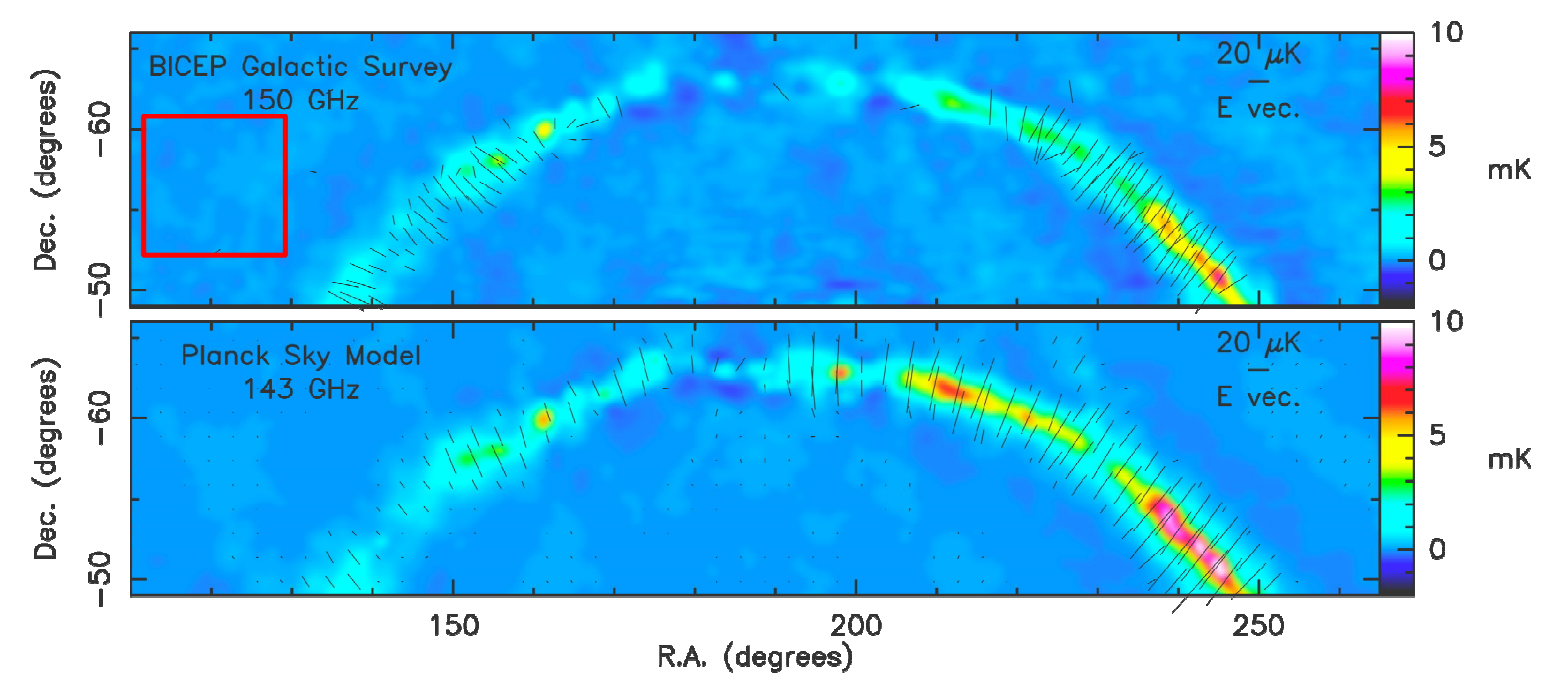

Figure 2. BICEP1 devotes $15 \%$ of each day to observing a region spanning the Galactic plane $\left(l=265^{\circ}\right.$ to $\left.340^{\circ}\right)$. The top figure shows that BICEP1 detects polarized emission along most of the Galactic plane, with a median polarization fraction of $2.0 \%$ in brighter regions $(\mathrm{T}>500 \mu \mathrm{K})$. The red box on the left shows a moderate Galactic latitude region in which BICEP1 has integrated deeply, and (after subtracting $\mathrm{CMB}$ ) detects unpolarized dust structure at the $50 \mu \mathrm{K}$ level similar to that in the FDS model (too faint to be visible on this color scale). Preliminary constraints on dust polarization in this region are $<5 \%$, consistent with recent WMAP all-sky estimates. The Planck consortium has begun comparing these BICEP maps to the detailed foreground predictions of the Planck Sky Model, a draft version of which is shown in the lower panel (courtesy G. Rocha/PSM team).

\section{THE TECHNICAL APPROACH}

\subsection{The plan}

Our technical approach is based on the proven concept from BICEP1 in the field. BICEP1 has been observing the CMB continuously since late February 2006. In June 2006, we published a description of its performance [3], reporting details of calibration, atmospheric noise rejection, optical design and characterization, observing efficiency, and initial temperature and polarization maps. The lessons learned from building, characterizing, and operating BICEP1 guide our program of upgrades. 

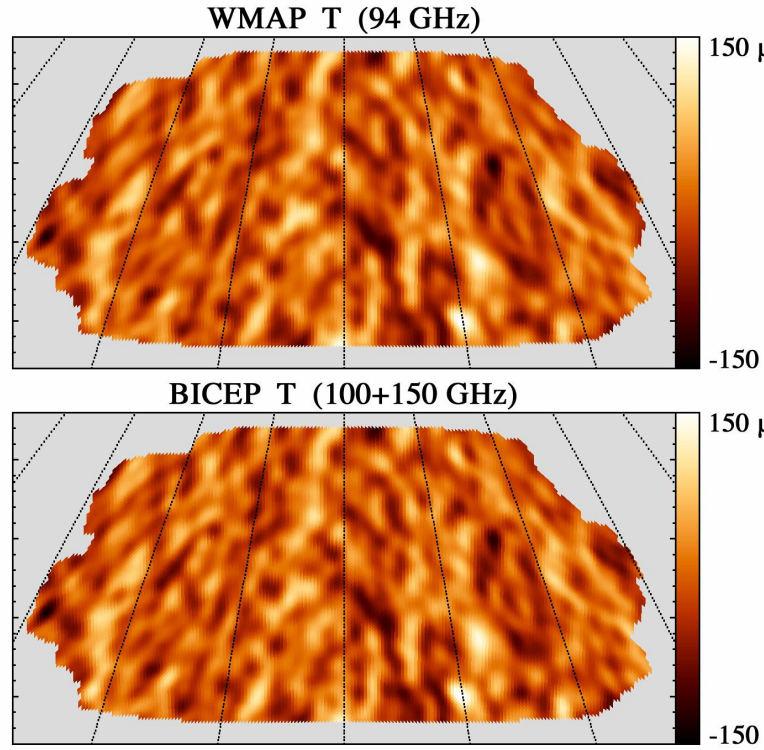

BICEP E $(100+150 \mathrm{GHz})$
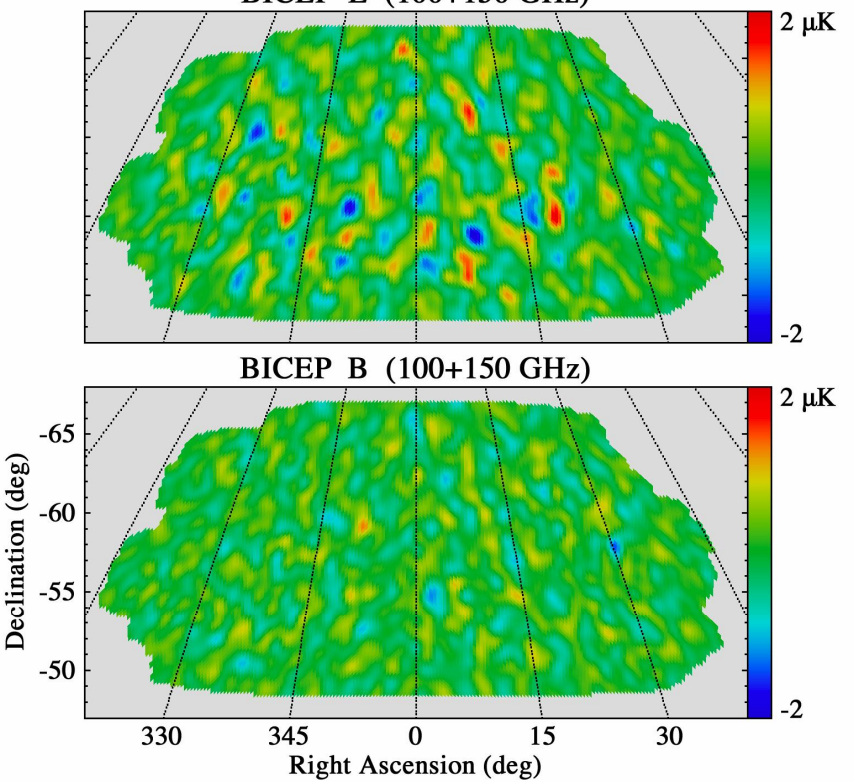

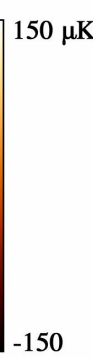

$150 \mu \mathrm{K}$

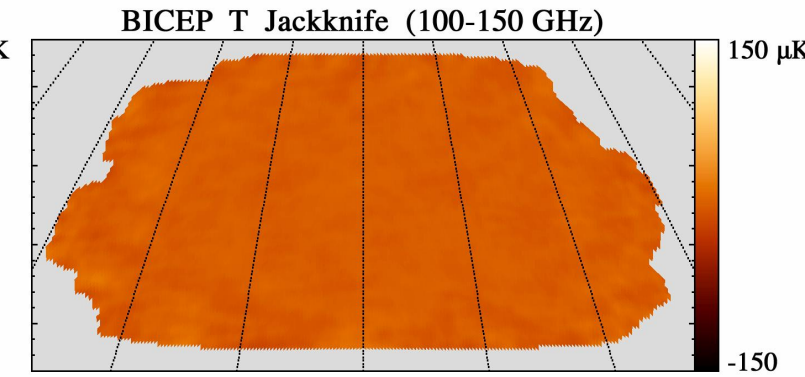

BICEP E Jackknife (100-150 GHz)

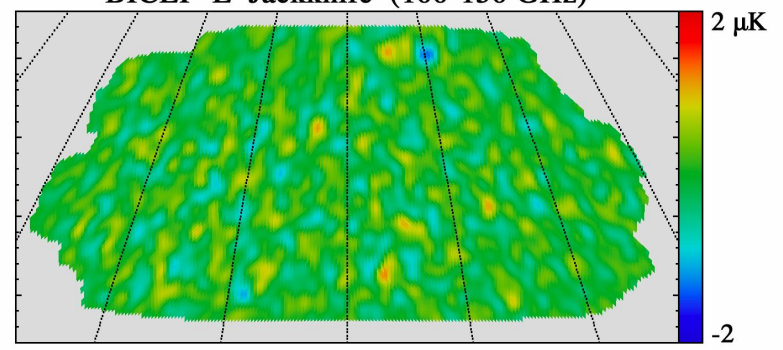

BICEP B Jackknife $(100-150 \mathrm{GHz})$

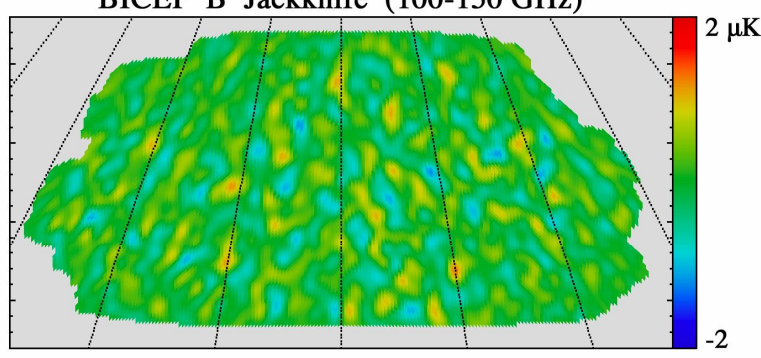

Figure 3. Maps of the BICEP1 CMB field including BICEP1 observations from February 2006 through 13 May 2007 , smoothed to 60' resolution. Top row: The WMAP W-band T map, filtered according to the BICEP1 observing strategy. The map shows total BICEP1 integration time (100 and $150 \mathrm{GHz})$ on this field, which in the central region exceeds 700,000 detector-seconds in each 1 $\mathrm{deg}^{2}$. 2nd row: BICEP1 T map. The excellent agreement with WMAP illustrates our ability to use the CMB temperature to derive a precise cross-calibration at each frequency. The temperature jackknife map, formed by subtracting the two BICEP1 frequencies, demonstrates the high signal-to-noise of the T maps and shows no evidence of foreground contamination in this field. Atmospheric fluctuations contribute a slight striping visible at large scales in the $\mathrm{T}$ jackknife map. Differencing polarized detectors removes these fluctuations, and after 1.3 seasons of integration the noise in BICEP1's Q and U jackknife maps is consistent with the measured detector NETs; the rms in $1 \mathrm{deg}^{2}$ pixels is $0.89 \mu \mathrm{K}$ for $100 \mathrm{GHz}$ and $0.77 \mu \mathrm{K}$ for $150 \mathrm{GHz}$. 3rd and 4th rows: BICEP1 Q and U maps are combined to produce Weiner-filtered E and B polarization maps. Both E and B jackknife maps and the B signal maps are consistent with noise. The E map shows degree-scale structure at the expected level.

Even on the large angular scales probed by BICEP, the Polar atmosphere is stable enough that high $\mathrm{S} / \mathrm{N}$ maps of degreescale CMB temperature anisotropy can be generated and compared with WMAP to perform absolute calibration on daily timescales. The residual atmospheric noise is subtracted virtually perfectly by the detector differences in the 
measurement of polarization; our current polarization maps achieve sensitivities consistent with the fundamental noise limits of our 98 detectors (Figure 3), showing E-modes at the expected level and no evidence of artifacts due to polarized atmosphere or ground pickup. The BICEP1 receiver was given a rigorous shakedown at Caltech prior to being deployed to South Pole. Since the aperture is small, the far-field beam patterns could be measured within the highbay lab using the mount to scan the telescope. Systematic errors due to beam asymmetries were measured to be low enough to permit integration to $r<0.1$ for BICEP1 [4].
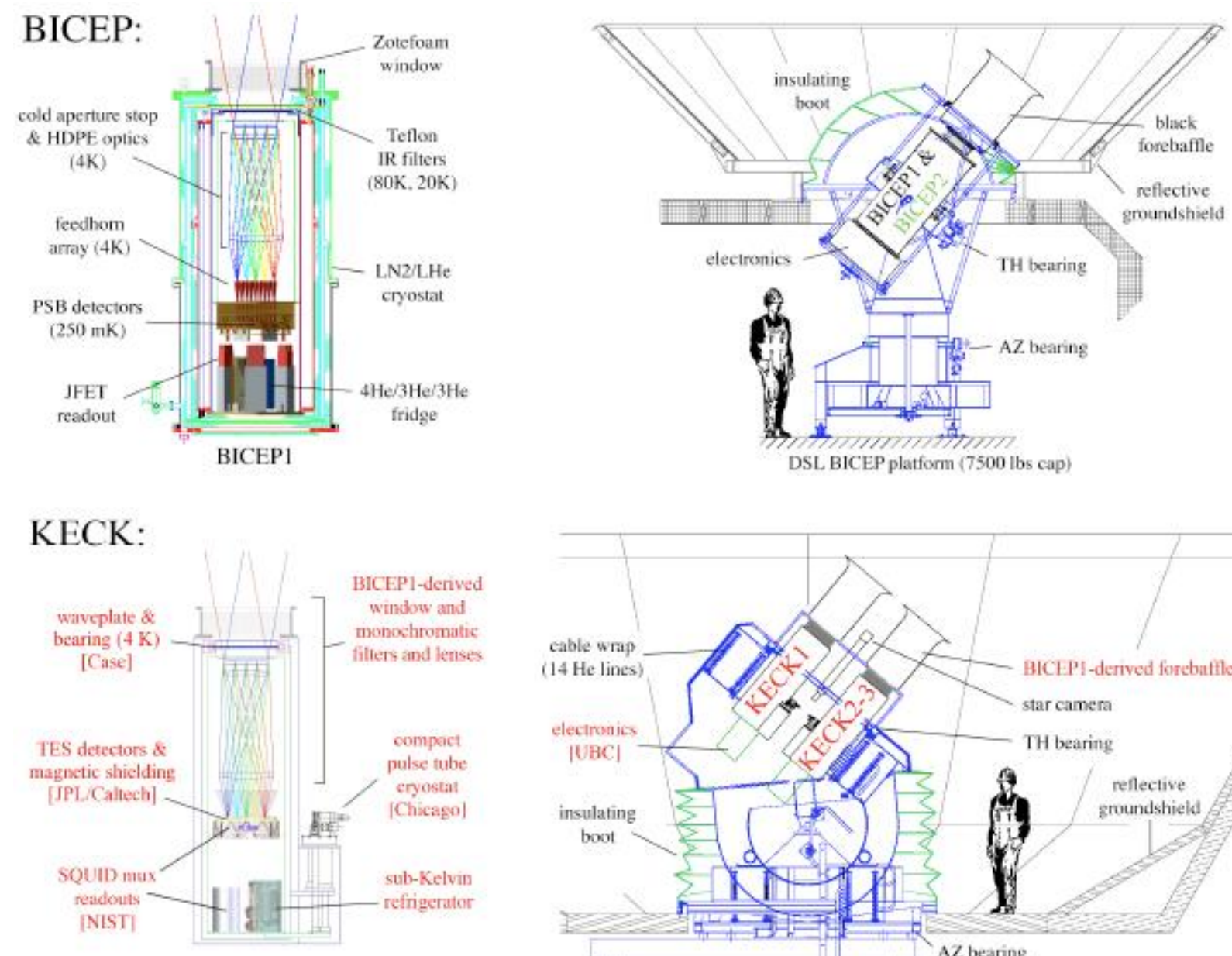

$\operatorname{KECK} 1,2,3$

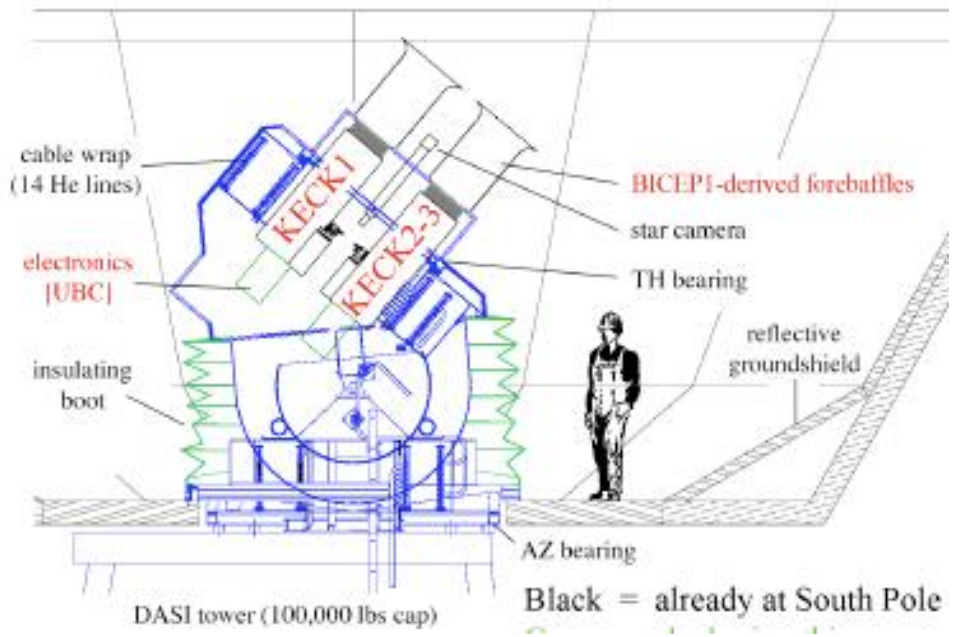

Figure 4. Top: The BICEP1 cryostat and optics and the BICEP mount. BICEP1's unique refracting optical design consists of two Teflon-AR-coated polyethylene lenses cooled to $4 \mathrm{~K}$ that produce a large, flat focal plane with a cold aperture stop at the $25 \mathrm{~cm}$ primary lens. Optics and detector insert are housed within a toroidal LN2/LHe cryostat, with a large, low-loss Zotefoam window and thermal blocking filters to accommodate the optical path. The compact BICEP mount points the receiver $(<10$ arcmin), and is capable of rapid scanning in azimuth $(4 / \mathrm{sec})$ and complete boresight rotation. The BICEP 2 receiver achieves higher sensitivity and improved control of systematics using monochromatic antenna-coupled TES detector arrays and a half-wave plate. It will demonstrate these upgrades in a LHe cryostat similar to that of BICEP1. Bottom: SPUD (tentatively relabeled here "The Keck Array") will house identical polarimeters operating at 100,150 , and $220 \mathrm{GHz}$ in compact, mechanically-cooled cryostats for deployment on the existing DASI mount. The larger and higher capacity DASI mount has similar pointing and scanning capabilities to the BICEP mount and is already plumbed for mechanical coolers. 


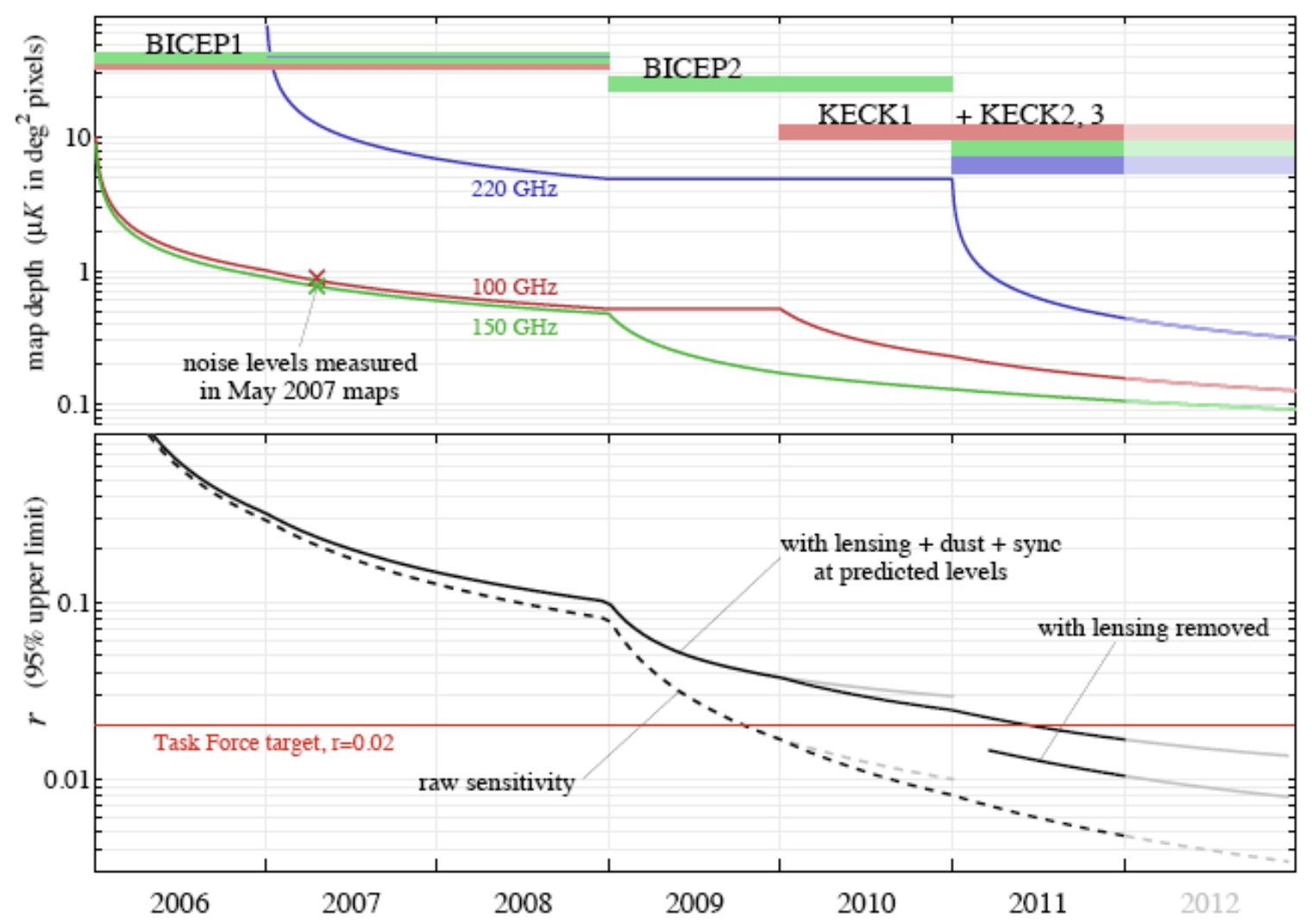

Figure 5. The phased program we are planning for BICEP1/BICEP2 and SPUD. Notice that the name SPUD has been replaced by KECK in this figure; we have recently submitted a proposal to Keck Foundation requesting funding for the SPUD receivers, and the proposal is currently under consideration. The top panel shows mapping depth in uK in the center of the 800 deg2 BICEP field, for the three observing frequencies. Although these are projected sensitivities, we note that thus far noise levels achieved in BICEP1 maps lie on these curves. The lower panel shows power to constrain inflation, giving 95\% upper limits on $\mathrm{r}$ under different foreground scenarios. The depth takes a steep dive after deploying BICEP2, and by the end of its first season reaches $r=0.03$. At this point the multi-frequency coverage of the SPUD (here "Keck Array") receivers becomes valuable, allowing sufficient foreground discrimination to push down to $r=0.01$.

As mentioned earlier, BICEP2/SPUD is a series of receivers designed to cover 3 separate frequency bands. BICEP2 is for $150 \mathrm{GHz}$ and will be replacing the BICEP1 receiver (austral summer 09), followed in subsequent years by SPUD (Keck) 1, 2 and 3 which are for 100, 150, and $220 \mathrm{GHz}$. BICEP2 is a liquid helium cryostat with vapor cooling shield (no liquid nitrogen). SPUDs are cryogen-free cryostats, equipped with pulse tube coolers made by $\mathrm{Cryomech}^{\circledR}$ in order to reach to $4 \mathrm{~K}$.

\subsection{Cryogenic receiver, optics and TES focal plane unit}

The 4K insert of each BICEP2/SPUD receiver is an assembly of two units: the camera tube and telescope tube. The camera tube (Figure 5) is supported by a $4 \mathrm{~K}$ coldplate and houses the $250 \mathrm{mK}$ cryocooler (closed cycle, three-staged ${ }^{4} \mathrm{He} /{ }^{3} \mathrm{He} /{ }^{3} \mathrm{He}$ cooler) and the FPU. The FPU is thermally isolated from the $4 \mathrm{~K}$ environment by means of a three-stage vespel truss; parasitic heat from $4 \mathrm{~K}$ stage is intercepted at $2 \mathrm{~K}$ and subsequently at $350 \mathrm{mK}$. The FPU are surrounded by superconducting lamp shade-shaped structure $(\mathrm{Nb})$ which provides extra magnetic shielding (Figure 6, Right).

The telescope tube is supported by the Camera tube and it houses the optical train. We adopt BICEP1 small aperture design (Figure 6, Left) which has been demonstrated to give several advantages: i. the system is highly efficient to assemble, test and deploy. ii. the small aperture offers excellent stability since the entire optics is at $4 \mathrm{~K}$. iii. the far field of a degree beam is typically in the range of $50 \mathrm{~m}$, allowing rigorous characterization of the instrument beam in the laboratory. iv. an aperture-filling cooled waveplate offers ideal polarization modulation, and v. a small forebaffle gives superior sidelobe rejection, as a result the analysis of BICEP1 data so far has not required ground subtraction. 

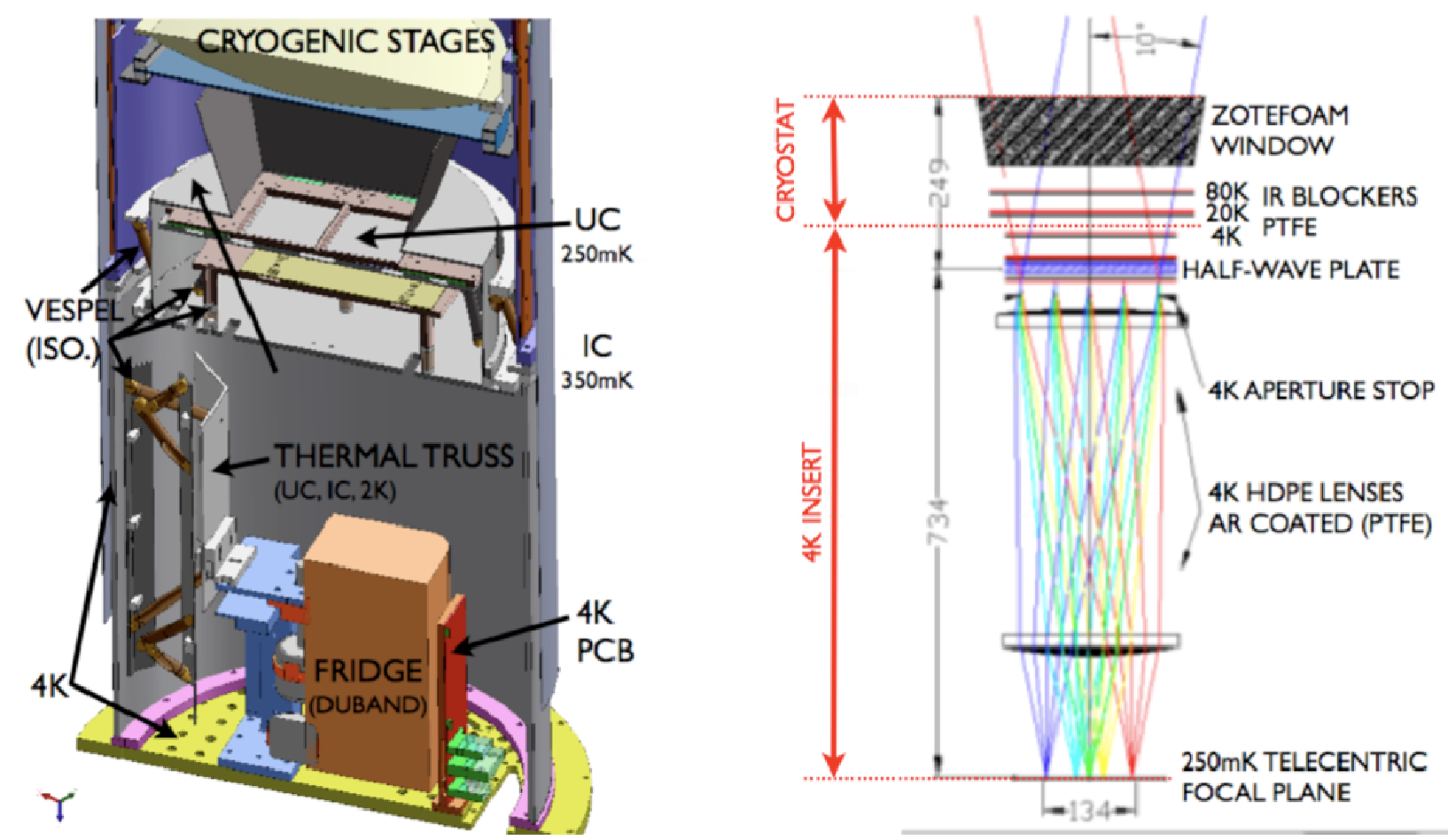

Figure 6. Left: The Camera Tube. Right: The Optical Design. BICEP2/SPUD optics follows the proven BICEP1's small aperture telecentric design, optimized for single frequency with stripline antenna coupled detectors. The design reduces absolute ellipticity by an order of magnitude, with symmetric aperture illumination to further mitigate exposure to polarization systemmatics.

The heart of the receiver is a 512 (256 dual-polarizations pixels, $\sim 5$ times more than BICEP1's) TES detector array, read out with NIST time-domain multiplexed SQUID amplifiers. The beams formed by the planar antennas couple directly to the optical system, eliminating additional fore-optics. Lithographic fabrication allows easy scaling to large array formats.

Each pixel comprises an array of dual-polarization slot antennas that couple incoming radiation to a pair of matched detectors[5]. The detectors in each pair can be differenced to reject common mode signals such as atmospheric emission, drifts in the multiplexed readout chain, or focal plane temperature fluctuations. The spectral band is defined on the focal plane using a lumped-element microstrip filter to give $25 \%$ bandwidth. We have developed a $150 \mathrm{GHz}$ array consisting of 64 such pixels on a single tile, with a complete focal plane comprising four tiles.

Measured characteristics of this array technology, shown in Figure 7, closely match theoretical predictions in key performance areas of beam pattern (ellipticity $<1 \%$ ), spectral band pass (no measurable leaks down to $-25 \mathrm{~dB}$ ), optical efficiency $(>70 \%)$, and noise performance. The observed efficiency is consistent with known small losses in the antenna.

To match the expected optical loading, we will operate the arrays from $250 \mathrm{mK}$ and use Titanium (Ti) TES films with a transition temperature at $450 \mathrm{mK}$. Because the Ti sensors will saturate on a $300 \mathrm{~K}$ background, we have developed an Aluminum (Al) TES with TC $=1.2 \mathrm{~K}$ in series with the Ti TES which allows end-to-end optical testing in a laboratory environment with high background loading. The $\mathrm{Al}$ films have a relatively large $0.5 \mathrm{~W}$ impedance to satisfy the stability condition of the SQUID readout. The required fabrication parameters, listed in Table 2, have all been achieved and verified in device testing.

Figure 7 shows the noise performance of a series Ti-Al TES biased at the $450 \mathrm{mK}$ Ti transition. The demonstrated stability (1/f knee $=40 \mathrm{mHz})$ enables us to operate with a stepped half-wave plate, retaining BICEP1's strategy of combined scanning and detector differencing as the primary modulation. The measured dark NEP of $2 \times 10-17 \mathrm{~W} / \mathrm{pHz}$ meets the requirement for BICEP2/SPUD. 
Figure 7. Top Left: A single 150 $\mathrm{GHz}$ pixel comprising a 288 element phased array of dual polarization slot antennas coupled to a matched load by a superconducting microstrip network. Microstrip band-defining filters and TES detectors are visible at bottom. Bottom Left: Measured beam pattern. The beam ellipticity is $<1 \%$. Polarization efficiency is $>98 \%$. The sidelobes $(<-13 \mathrm{~dB})$ will be truncated at the $4 \mathrm{~K}$ primary aperture stop. Top right: Measured spectra - orange and blue curves correspond to unfiltered and filtered antenna spectral response. Bottom right: Measured electrical NEP of a Ti TES. More on this can be found in Kuo et al. in this proceeding[5].
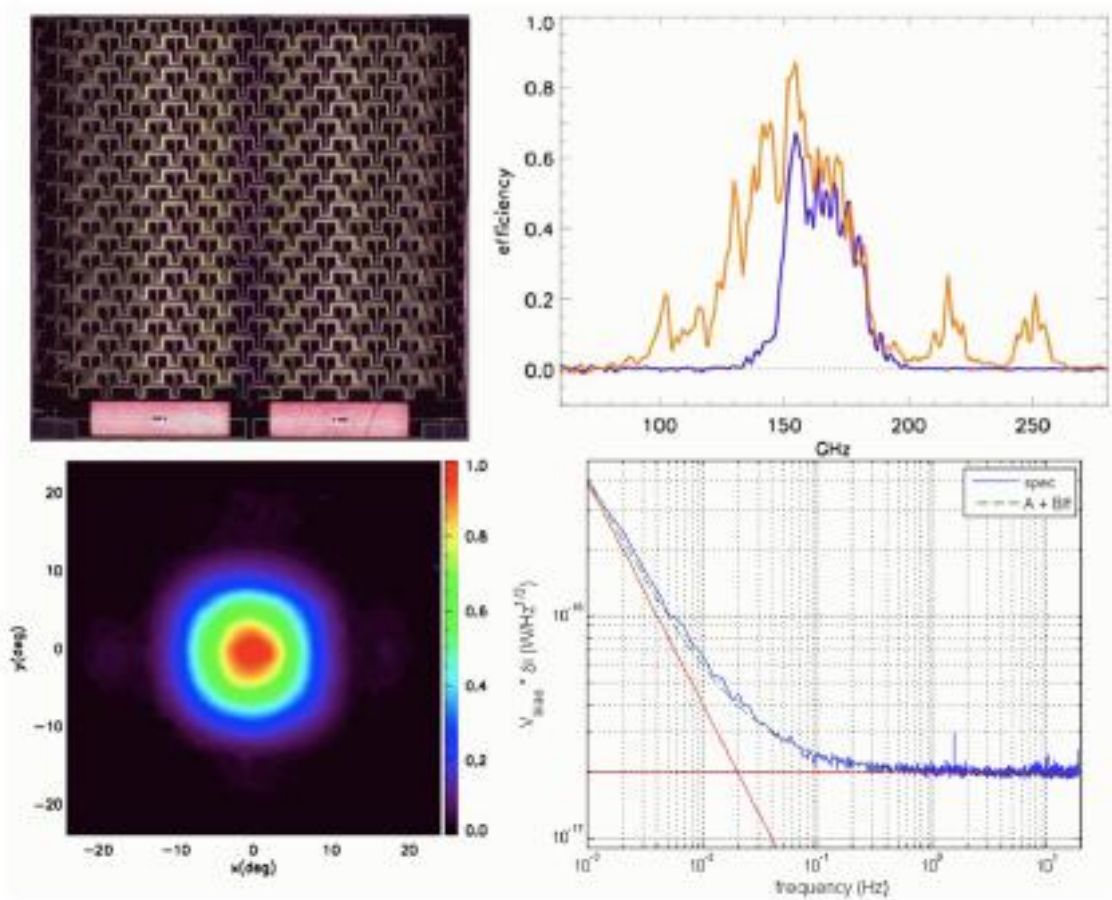
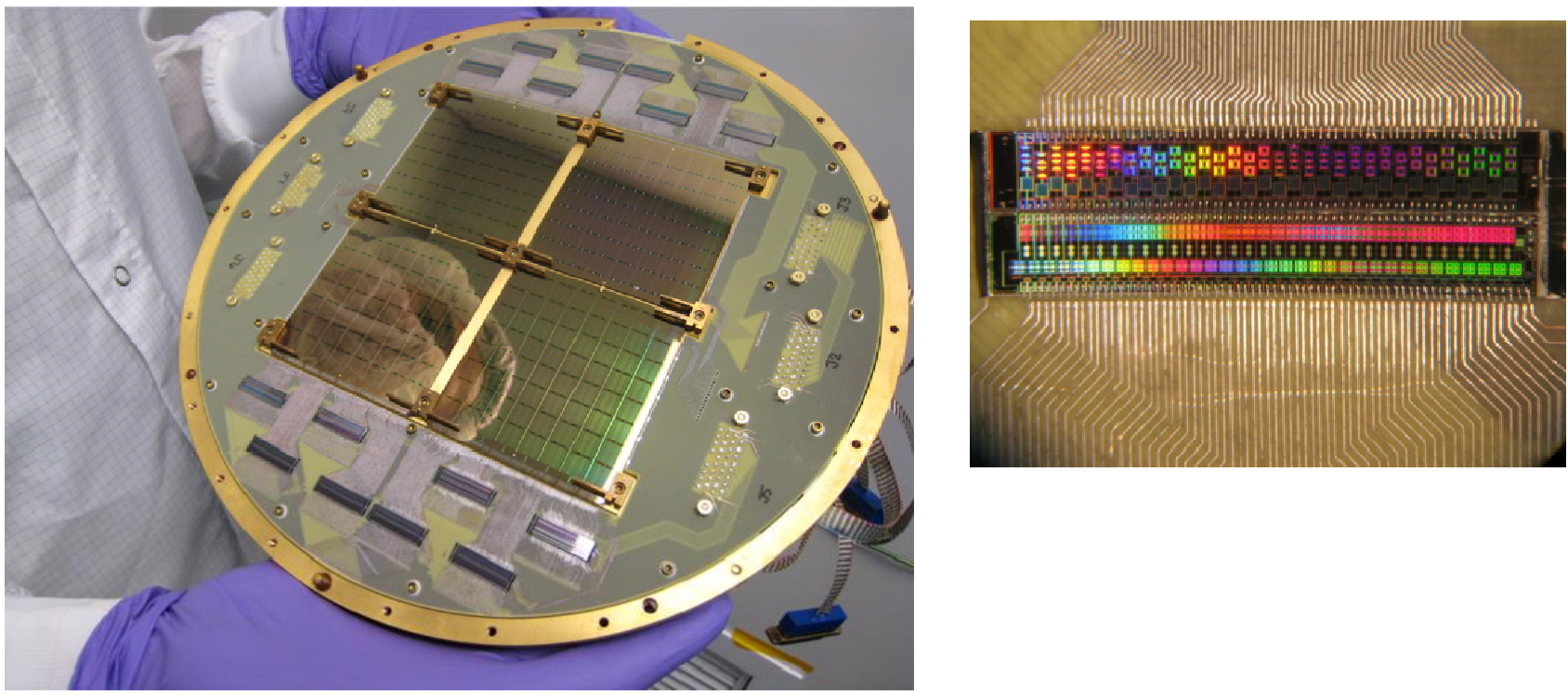

Figure 8. Left: BICEP2 Antenna-coupled TES detector arrays, with 256 dual-polarization pixels. The four tile arrays are firmly mounted on an OFHC copper plate, and the signals are routed to Nyquist/Squid Mux chips and subsequently to the 4K squid readout via $\mathrm{Al}$ wirebonds and traces. The assembly is completed with a superconducting $\mathrm{Nb}$ backshort to provide extra magnetic shielding. The FPU is magnetic free and modular, and carefully designed to minimize flux trap. Right: Nyquist/Squid Mux chip (NIST).

Each focal plane will consist of 4 array tiles illuminated with an $\mathrm{f} / 3$ beam, giving $2 \mathrm{f} \lambda$ pixels with $-12 \mathrm{~dB}$ edge taper on the cold aperture stop at the primary lens (Figure 8). This packing density is nearly ideal for CMB measurements. The detector arrays and SQUIDs are integrated in a simple and robust structure. The 4 arrays in the focal plane are mated to a 
circuit board with superconducting Al wirebonds, and connected to discrete 32:1 SQUID and Nyquist chips mounted on the perimeter.

\subsection{Magnetic Shielding}

Unlike BICEP1 which used NTD bolometers and JFET readout, BICEP2/SPUD instruments will use TESs and SQUIDs readout, and thus require careful considerations for magnetic shielding. A SQUID, of course, measures current via the magnetic field in its input inductor coil. TES bolometers are also sensitive to magnetic field through the dependence of Tc on applied field. Fortunately, these susceptibilities are well characterized in the lab so one can specify the magnetic shielding and then develop an optimal design.

For magnetic shielding, we incorporate a single layer of cryoperm at $4 \mathrm{~K}$ and $250 \mathrm{mK} \mathrm{Nb}$ box with a flared opening through which light enters, and a $\mathrm{Nb}$ plane just under the array. With $50 \mu \mathrm{T}$ applied field at a $45^{\circ}$ angle to the optical axis and, our model yields a residual field of a few parts in $10^{6}$ over most of the focal plane.

In addition, NIST has successfully developed and tested next-generation 32:1 SQUID multiplexer chips using gradiometer-wound coils to reduce B-field susceptibility by a factor of $\sim 100$ compared to the previous design. Based on this much-reduced susceptibility and TES's inherent sensitivity to B-field, we estimate the scan-synchronous magnetic field signal due to earth's field must be attenuated by $10^{8}$ for the SQUIDs and $10^{6}$ for the TESs to ensure such signals are $<5 \%$ of the target B-mode signal on degree scales. We meet this design goal with a combination of high-permeability and superconducting magnetic shields. In actual observations, common-mode signal rejection and averaging effects provide significant additional safety margin for rejection of magnetic field pickup.

\section{SUMMARY}

BICEP2 and SPUD are phased programs based on the proven concept of BICEP1, designed to detect the B-mode signal of polarization at level of $r=0.01$ targeted by the CMB 2006 Task Force. We have completed all the major hardware milestones for BICEP2, the first experiment in the series, including the design, fabrication and integration of the cryogenic receiver and TES focal plane, optics and magnetic shielding. We have completed our cryogenic tests. Dark and optical tests of the integrated receiver are underway. Our goal is to rigorously and completely characterize the receiver, including the instrument sensitivity and optical performance. These tests include mounting BICEP2 receiver on the exact replica of BICEP telescope mount and assessing optical performance and the system sensitivity in deep integrations replicating the scanning strategy used in the field. BICEP2 will be deployed to the South Pole upon completion of this test program. Funding has been granted through NSF OPP-0742818 for BICEP2 operations at the South Pole through 2010, and for SPUD operations through 2011.

\section{REFERENCES}

[1] Spergel, D., "Exploring the cosmic background radiation with WMAP," this proceeding.

[2] R. Weiss et al. 2006, "Task Force on Cosmic Microwave Background Radiation", Report to NSF/NASA/DOE, astro-ph 0604101.

[3] Yoon, K. et al., "The Robinson Gravitational Wave Background Telescope (BICEP): a bolometric large angular scale CMB polarimeter," Proc. SPIE 6275, pp. 62751K (2006).

[4] Takahashi, Y. et al., "CMB polarimetry with BICEP: instrument characterization, calibration, and performance," this proceeding.

[5] Kuo, C.L. et al., “Antenna-coupled TES arrays for CMB polarimetry,” this proceeding.

[6] Kuo, C.L. et al., "Antenna-Coupled TES Bolometers for CMB Polarimetry”, Proc. SPIE 6275, pp. 62751M (2006). 